\title{
Argument Properties for a Class of Analytic Functions Involving Libera Transform
}

\author{
Badr S. Alkahtani, ${ }^{1}$ Teodor Bulboacă, ${ }^{2}$ Rakesh Kumar, ${ }^{3}$ and Rubayyi Alqahtanii ${ }^{4}$ \\ ${ }^{1}$ Mathematics Department, College of Science, King Saud University, Riyadh 11989, Saudi Arabia \\ ${ }^{2}$ Faculty of Mathematics and Computer Science, Babeş-Bolyai University, 400084 Cluj-Napoca, Romania \\ ${ }^{3}$ Department of Mathematics, Amity University Rajasthan, NH-11C, Jaipur 302002, India \\ ${ }^{4}$ Department of Mathematics and Statistics, College of Science, Al-Imam Mohammad Ibn Saud Islamic University (IMSIU), \\ P.O. Box 65892, Riyadh 11566, Saudi Arabia
}

Correspondence should be addressed to Rakesh Kumar; rkyadav11@gmail.com

Received 10 June 2016; Accepted 4 September 2016

Academic Editor: Jaeyoung Chung

Copyright (C) 2016 Badr S. Alkahtani et al. This is an open access article distributed under the Creative Commons Attribution License, which permits unrestricted use, distribution, and reproduction in any medium, provided the original work is properly cited.

The purpose of this paper is to find sufficient argument properties, such that the images of some subclasses of functions by the Libera transform have bounded arguments.

\section{Introduction}

Let $\mathscr{A}$ denote the class of functions of the form

$$
f(z)=z+\sum_{n=2}^{\infty} a_{n} z^{n}
$$

which are analytic in the open unit disk $\mathscr{U}=\{z \in \mathbb{C}:|z|<1\}$.

A function $f \in \mathscr{A}$ is said to be in the class $\mathcal{S}^{*}(\alpha)$ of starlike functions of order $\alpha$ in $\mathcal{U}$, if and only if it satisfies the condition

$$
\operatorname{Re} \frac{z f^{\prime}(z)}{f(z)}>\alpha, \quad z \in \mathcal{U},(\alpha<1) .
$$

A function $f \in \mathscr{A}$ is said to be in the class $\mathscr{K}^{*}(\alpha)$ of convex functions of order $\alpha$ in $\mathcal{U}$, if and only if it satisfies the condition

$$
\operatorname{Re}\left(1+\frac{z f^{\prime \prime}(z)}{f^{\prime}(z)}\right)>\alpha, \quad z \in \mathscr{U},(\alpha<1) .
$$

It is well-known that $\mathcal{S}^{*}(\alpha) \subset \mathcal{S}^{*}, \mathscr{K}(\alpha) \subset \mathscr{K}$ whenever $0 \leq \alpha<1$, where $\mathcal{S}^{*} \equiv \mathcal{S}^{*}(0)$ and $\mathscr{K} \equiv \mathscr{K}(0)$ represent, respectively, the class of starlike and convex (normalized) functions.
If $f \in \mathscr{A}$ satisfies the condition

$$
\operatorname{Re} \frac{f(z)}{z f^{\prime}(z)}>\beta, \quad z \in \mathcal{U},(0 \leq \beta<1),
$$

then $f$ is said to be star-like of reciprocal order $\beta$, and we denote this class by $\mathcal{N} \mathcal{S}^{*}(\beta)$.

The above definition was recently discussed by Nunokawa et al. [1] and Ravichandran and Sivaprasad Kumar [2].

Motivated by their works, we define a class of convex functions of reciprocal order $\beta$ as follows.

Definition 1. If $f \in \mathscr{A}$ satisfies the condition

$$
\operatorname{Re}\left(1+\frac{z f^{\prime \prime}(z)}{f^{\prime}(z)}\right)^{-1}>\beta, \quad z \in \mathcal{U},(0 \leq \beta<1),
$$

then $f$ is said to be convex function of reciprocal order $\beta$, and we denote this class by $\mathscr{N} \mathscr{K}(\beta)$.

Definition $2($ see $[3,4])$. Suppose that $f$ and $g$ are two analytic functions in $\mathcal{U}$. We say that the function $f$ is subordinate to $g$, written $f(z) \prec g(z)$, if there exists a Schwarz function $w$, that is, a function $w$ analytic in $\mathscr{U}$, with $w(0)=0$ and $|w(z)|<1$, such that $f(z)=g(w(z)), z \in \mathscr{U}$. 
It is well-known that (see, e.g., $[3,4])$ if $g$ is univalent in $\mathcal{U}$, then the following subordination property holds:

$$
\begin{gathered}
f(z) \prec g(z) \Longleftrightarrow \\
f(0)=g(0), \\
f(\mathcal{U}) \subset g(\mathcal{U}) .
\end{gathered}
$$

Remarks 1. (1) In view of the fact that

$$
\begin{aligned}
& \operatorname{Re} p(z)>\beta, \quad z \in \mathcal{U}, \quad(0 \leq \beta<1) \\
& \Downarrow \\
& \quad \operatorname{Re} \frac{1}{p(z)}=\operatorname{Re} \frac{p(z)}{|p(z)|^{2}}>0, \quad z \in \mathcal{U},
\end{aligned}
$$

it follows that a star-like (convex) function of reciprocal order $\beta$ is a star-like (convex) function. Thus,

$$
\begin{aligned}
& \mathcal{N} \mathcal{S}^{*}(\beta) \subset \mathcal{S}^{*}(0), \\
& \mathcal{N} \mathscr{K}(\beta) \subset \mathscr{K}(0),
\end{aligned}
$$

$$
(0 \leq \beta<1)
$$

and the equality holds in both cases if and only if $\beta=0$.

(2) Let $0<\beta<1$, and suppose that $f \in \mathscr{A}$. Then

(i)

$$
\begin{array}{r}
f \in \mathcal{N} \mathcal{S}^{*}(\beta) \Longleftrightarrow \\
\left|\frac{z f^{\prime}(z)}{f(z)}-\frac{1}{2 \beta}\right|<\frac{1}{2 \beta}, \quad z \in \mathcal{U},
\end{array}
$$

(ii)

$$
\begin{array}{r}
f \in \mathcal{N} \mathscr{K}(\beta) \Longleftrightarrow \\
\left|1+\frac{z f^{\prime \prime}(z)}{f^{\prime}(z)}-\frac{1}{2 \beta}\right|<\frac{1}{2 \beta}, \quad z \in \mathcal{U} .
\end{array}
$$

(3) We note that, for $0 \leq \beta<1$, we have

$$
\begin{aligned}
f \in \mathcal{N} \mathscr{K}(\beta) & \Longleftrightarrow \\
z f^{\prime}(z) \in \mathcal{N} \mathcal{S}^{*}(\beta) . &
\end{aligned}
$$

In the following, we give some examples of functions belonging to the class of star-like functions of reciprocal order and the class of convex functions of reciprocal order.

Example 3. (1) For $0 \leq \beta<1$, define the function $f_{\beta}: \mathcal{U} \rightarrow \mathbb{C}$ by

$$
\begin{aligned}
& f_{\beta}(z) \\
& = \begin{cases}z[1+(1-2 \beta) z]^{-2(1-\beta) /(1-2 \beta)}, & \text { if } \beta \in[0,1) \backslash\left\{\frac{1}{2}\right\}, \\
z e^{-z}, & \text { if } \beta=\frac{1}{2} .\end{cases}
\end{aligned}
$$

Since $f_{\beta} \in \mathscr{A}$ and a simple computation shows that

$$
\operatorname{Re} \frac{f_{\beta}(z)}{z f_{\beta}^{\prime}(z)}=\operatorname{Re} \frac{1+(1-2 \beta) z}{1-z}>\beta, \quad z \in \mathcal{U},
$$

we conclude that $f_{\beta} \in \mathcal{N} \mathcal{S}^{*}(\beta)$.

(2) Using the third part of the Remarks 1, we deduce that

$$
\begin{gathered}
\varphi_{\beta} \in \mathcal{N} \mathscr{K}(\beta) \Longleftrightarrow \\
f_{\beta}(z)=z \varphi_{\beta}^{\prime}(z) \in \mathscr{N} \mathcal{S}^{*}(\beta) .
\end{gathered}
$$

Since we proved that $f_{\beta} \in \mathcal{N} \mathcal{S}^{*}(\beta)$, and from the above differential equation we obtain

$$
\begin{aligned}
& \varphi_{\beta}(z) \\
& = \begin{cases}\int_{0}^{z}[1+(1-2 \beta) u]^{-2(1-\beta) /(1-2 \beta)} d u, & \text { if } \beta \in[0,1) \backslash\left\{\frac{1}{2}\right\}, \\
1-e^{-z}, & \text { if } \beta=\frac{1}{2},\end{cases}
\end{aligned}
$$

it follows that $\varphi_{\beta} \in \mathcal{N} \mathscr{K}(\beta)$.

Example 4. (1) For $1 / 2 \leq \beta<1$, consider the function $\psi_{\beta}$ : $\mathcal{U} \rightarrow \mathbb{C}$, defined by

$$
\psi_{\beta}(z)=z e^{((1-\beta) / \beta) z}
$$

This gives that

$$
\operatorname{Re} \frac{\psi_{\beta}(z)}{z \psi_{\beta}^{\prime}(z)}=\operatorname{Re} \frac{\beta}{\beta+(1-\beta) z}=\operatorname{Re} h(z), \quad z \in \mathcal{U},
$$

where

$$
h(z)=\frac{\beta}{\beta+(1-\beta) z} .
$$

Since $\operatorname{Re} \beta \geq 1 / 2$, it follows that $h$ is analytic in $\mathcal{U}$. Using the fact that $h$ is a convex (not necessary normalized) function in $\mathcal{U}$ and $h(\mathcal{U})$ is symmetric with respect to the real axis, we deduce that $\operatorname{Re} h(z)>\beta$ for all $z \in \mathcal{U}$; hence $\psi_{\beta} \in \mathcal{N} \mathcal{S}^{*}(\beta)$.

(2) From the above result, using the same reasons like in the second part of Example 3, we obtain that

$$
\begin{aligned}
& \chi_{\beta}(z)=\int_{0}^{z} e^{((1-\beta) / \beta) u} d u \in \mathscr{N} \mathscr{K}(\beta), \\
& \qquad\left(\frac{1}{2} \leq \beta<1\right) .
\end{aligned}
$$

We mention that several authors have investigated the strongly star-like functions and the strongly convex functions (see [5-18]). In the present investigation we give some argument properties of analytic functions belonging to $\mathscr{A}$, such that the images of these functions by the Libera transform have bounded arguments. 


\section{Main Results}

The following lemma will be used to prove our main results.

Lemma 5 (see [16]). Let $\alpha_{0}$ be the solution of

$$
\alpha \pi=\frac{3 \pi}{2}-\tan ^{-1} \gamma
$$

for a suitable fixed $\gamma>0$, so that $\lambda: \mathcal{U} \rightarrow \mathbb{C}$ satisfies

$$
\frac{\alpha \operatorname{Re} \lambda(z)}{1+\alpha|\operatorname{Im} \lambda(z)|} \geq \gamma, \quad z \in \mathcal{U},
$$

and let

$$
\eta:=\eta(\alpha, \gamma)=\alpha+\frac{2}{\pi} \tan ^{-1} \gamma, \quad 0<\alpha \leq \alpha_{0} .
$$

If $p$ is analytic in $\mathcal{U}, p(0)=1$ and

$$
\left|\arg \left[p(z)+\lambda(z) z p^{\prime}(z)\right]\right|<\frac{\pi}{2} \eta, \quad z \in \mathcal{U},
$$

then

$$
|\arg p(z)|<\frac{\pi}{2} \alpha, \quad z \in \mathcal{U}
$$

Remark that in the article [19] the author considered some special situations improving many results with a lot of applications. Thus, in [19, Lemma 2], the author proved the next result.

Let $\lambda$ be a function defined on $\mathcal{U}$ satisfying

$$
\begin{aligned}
|\arg (\lambda(z)-\eta)| & <\frac{\pi}{3}, \quad z \in \mathcal{U}, \\
\text { such that } \eta & =\inf _{z \in \mathscr{U}}\left(\operatorname{Re} \lambda(z)-\frac{|\operatorname{Im} \lambda(z)|}{\sqrt{3}}\right),
\end{aligned}
$$

and let

$$
\begin{aligned}
\beta^{\prime}(\eta)= & \left(\frac{6+5 \eta^{2}+2 \sqrt{9+15 \eta^{2}}}{25 \eta^{2}}\right)^{1 / 3} \\
& \cdot \frac{9-2 \sqrt{9+15 \eta^{2}}}{10}
\end{aligned}
$$

be such that $2 \beta^{\prime}(\eta)+\eta \geq 0$. If $p$ is analytic in $\mathcal{U}, p(0)=1$, then

$$
\begin{aligned}
& \operatorname{Re}\left[p(z)+\lambda(z) z p^{\prime}(z)\right]>\beta^{\prime}(\eta), \quad z \in \mathcal{U} \\
& \Downarrow \\
&|\arg p(z)|<\frac{\pi}{3}, \quad z \in \mathcal{U} .
\end{aligned}
$$

We emphasize that a special case of this lemma improves the conclusion of Lemma 1 from [19], and in the same article the author derived a number of interesting consequences of it.
Let $\mathscr{L}: \mathscr{A} \rightarrow \mathscr{A}$ defined by

$$
\mathscr{L} f(z)=\frac{2}{z} \int_{0}^{z} f(t) d t
$$

be the well-known Libera transform [20].

We first prove the following theorem, which is essential for proving our other results.

Theorem 6. For $g \in \mathscr{A}$ suppose that the Libera transform $G=$ $\mathscr{L} \mathrm{g}$ satisfies the condition (21), with

$$
\lambda(z)=\left(2+\frac{z G^{\prime \prime}(z)}{G^{\prime}(z)}\right)^{-1},
$$

where $0<\alpha \leq \alpha_{0}$ and $\alpha_{0}$ is defined by (20). If $f \in \mathscr{A}$ and $F=\mathscr{L} f$, then

$$
\left|\arg \frac{f^{\prime}(z)}{g^{\prime}(z)}\right|<\frac{\pi}{2} \eta(\alpha, \gamma)=\frac{\pi}{2}\left(\alpha+\frac{2}{\pi} \tan ^{-1} \gamma\right),
$$

$$
z \in \mathscr{U}
$$

implies

$$
\left|\arg \frac{F^{\prime}(z)}{G^{\prime}(z)}\right|<\frac{\pi}{2} \alpha, \quad z \in \mathcal{U} .
$$

Proof. Let us define the function $p$ by

$$
p(z)=\frac{F^{\prime}(z)}{G^{\prime}(z)}, \quad z \in \mathcal{U} \text {. }
$$

Suppose that there exists a number $z_{0} \in \mathcal{U}$, such that $G^{\prime}\left(z_{0}\right)=$ 0 ; since $G^{\prime}(0)=1$, then $z_{0} \in \mathcal{U} \backslash\{0\}$. It follows that there exists a unique number $n \in \mathbb{N}$ and a unique function $Q$, analytic in $\mathcal{U}$, with $Q\left(z_{0}\right) \neq 0$, such that

$$
G^{\prime}(z)=\left(z-z_{0}\right)^{n} Q(z), \quad z \in \mathcal{U} .
$$

From the above relation we deduce that

$$
\frac{z G^{\prime \prime}(z)}{G^{\prime}(z)}=\frac{n z}{z-z_{0}}+\frac{z Q^{\prime}(z)}{Q(z)}, \quad z \in \mathcal{U} \backslash\left\{z_{0}\right\},
$$

which implies that $z_{0}$ is a simple pole for the function $z G^{\prime \prime}(z) / G^{\prime}(z)$. Consequently, from (29) we obtain that $\lambda\left(z_{0}\right)=0$, and using the fact that $\gamma$ satisfies the inequality (21) for all $z \in \mathcal{U}$ it follows that

$$
\gamma \leq \frac{\alpha \operatorname{Re} \lambda\left(z_{0}\right)}{1+\alpha\left|\operatorname{Im} \lambda\left(z_{0}\right)\right|}=0,
$$

which contradicts the assumption $\gamma>0$.

Thus, $G^{\prime}(z) \neq 0$ for all $z \in \mathscr{U}$, which implies that the function $p$ is analytic in $\mathscr{U}$, and $p(0)=1$.

Now, a simple calculus shows that

$$
\frac{f^{\prime}(z)}{g^{\prime}(z)}=\frac{z F^{\prime \prime}(z)+2 F^{\prime}(z)}{z G^{\prime \prime}(z)+2 G^{\prime}(z)}=p(z)+\lambda(z) z p^{\prime}(z),
$$

and our result follows immediately from Lemma 5. 
Taking $g(z)=z$ in Theorem 6 , for the function $\lambda$ defined by (29) we have $\lambda(z)=1 / 2$, and we obtain the following result.

Corollary 7. Suppose that the parameters $\alpha$ and $\gamma$ satisfy the conditions (20) and (22), for a given $\gamma \leq \alpha / 2$. If $f \in \mathscr{A}$ and $F=\mathscr{L} f ;$ then

$$
\left|f^{\prime}(z)\right|<\frac{\pi}{2} \eta(\alpha, \gamma)=\frac{\pi}{2}\left(\alpha+\frac{2}{\pi} \tan ^{-1} \gamma\right), \quad z \in \mathscr{U}
$$

implies

$$
\left|\arg F^{\prime}(\mathrm{z})\right|<\frac{\pi}{2} \alpha, \quad z \in \mathcal{U} .
$$

Example 8. For $f \in \mathscr{A}$ and $F=\mathscr{L} f$, considering the special cases $\gamma=1 / \sqrt{3}, \gamma=\sqrt{2}-1$ and $\gamma=2-\sqrt{3}$, from the above corollary we obtain respectively the next implications:

(1) If $2 / \sqrt{3} \leq \alpha \leq 4 / 3$, then

$$
\begin{aligned}
& \left|f^{\prime}(z)\right|<\frac{\pi}{2} \eta\left(\alpha, \frac{1}{\sqrt{3}}\right)=\frac{\pi}{2}\left(\alpha+\frac{1}{3}\right), \quad z \in \mathscr{U} \\
& \Downarrow
\end{aligned}
$$

$\left|\arg F^{\prime}(z)\right|<\frac{\pi}{2} \alpha, \quad z \in \mathcal{U}$.

(2) If $2(\sqrt{2}-1) \leq \alpha \leq 11 / 8$, then

$$
\begin{gathered}
\left|f^{\prime}(z)\right|<\frac{\pi}{2} \eta(\alpha, \sqrt{2}-1)=\frac{\pi}{2}\left(\alpha+\frac{1}{4}\right), \quad z \in \mathscr{U} \\
\Downarrow
\end{gathered}
$$

$\left|\arg F^{\prime}(z)\right|<\frac{\pi}{2} \alpha, \quad z \in \mathscr{U}$.

(3) If $2(2-\sqrt{3}) \leq \alpha \leq 17 / 12$, then

$$
\begin{aligned}
\left|f^{\prime}(z)\right| & <\frac{\pi}{2} \eta(\alpha, 2-\sqrt{3})=\frac{\pi}{2}\left(\alpha+\frac{1}{6}\right), \quad z \in \mathscr{U} \\
& \Downarrow
\end{aligned}
$$

$\left|\arg F^{\prime}(z)\right|<\frac{\pi}{2} \alpha, \quad z \in \mathcal{U}$.

Theorem 9. For $g \in \mathscr{A}$ suppose that the Libera transform $G=$ $\mathscr{L} g \in \mathscr{N} \mathscr{K}(\beta), 0<\beta<1$. Suppose that $\alpha_{0}$ is defined by (20), and for $0<\alpha \leq \alpha_{0}$ let $\gamma$ satisfy the condition

$$
\begin{aligned}
0 & <\gamma \leq \tilde{\gamma}:=\tilde{\gamma}(\alpha, \beta) \\
& =\frac{2 \alpha \beta}{1+2 \beta+\sqrt{(\beta /(1+\beta)) \alpha^{2}+1}} .
\end{aligned}
$$

If $f \in \mathscr{A}$ and $F=\mathscr{L} f$, then

$$
\left|\arg \frac{f^{\prime}(z)}{g^{\prime}(z)}\right|<\frac{\pi}{2} \eta(\alpha, \gamma)=\frac{\pi}{2}\left(\alpha+\frac{2}{\pi} \tan ^{-1} \gamma\right),
$$

implies

$$
\left|\arg \frac{F^{\prime}(z)}{G^{\prime}(z)}\right|<\frac{\pi}{2} \alpha, \quad z \in \mathscr{U} .
$$

Proof. Let $p$ be the function defined by

$$
p(z)=\frac{F^{\prime}(z)}{G^{\prime}(z)}, \quad z \in \mathcal{U} .
$$

Similarly like in the proof of Theorem 6, we will prove that the function $p$ is analytic in $\mathcal{U}$. Supposing that there exists a number $z_{0} \in \mathcal{U}$, such that $G^{\prime}\left(z_{0}\right)=0$, since $G^{\prime}(0)=1$, then $z_{0} \in \mathscr{U} \backslash\{0\}$. Hence, there exists a unique number $n \in \mathbb{N}$ and a unique function $Q$, analytic in $\mathscr{U}$, with $Q\left(z_{0}\right) \neq 0$, such that

$$
G^{\prime}(z)=\left(z-z_{0}\right)^{n} Q(z), \quad z \in \mathcal{U} .
$$

From here we deduce that

$$
\frac{z G^{\prime \prime}(z)}{G^{\prime}(z)}=\frac{n z}{z-z_{0}}+\frac{z Q^{\prime}(z)}{Q(z)}, \quad z \in \mathscr{U} \backslash\left\{z_{0}\right\},
$$

which implies that $z_{0}$ is a simple pole for the function $z G^{\prime \prime}(z) / G^{\prime}(z)$. Now, using the fact that $G=\mathscr{L} g \in \mathscr{N} \mathscr{K}(\beta)$, we obtain

$$
0=\operatorname{Re}\left(1+\frac{z G^{\prime \prime}\left(z_{0}\right)}{G^{\prime}\left(z_{0}\right)}\right)^{-1}>\beta
$$

which contradicts the assumption $0 \leq \beta$.

Consequently, $G^{\prime}(z) \neq 0$ for all $z \in \mathcal{U}$, and this implies that the function $p$ is analytic in $\mathcal{U}$, with $p(0)=1$.

From (45) we easily get

$$
\frac{f^{\prime}(z)}{g^{\prime}(z)}=p(z)+\lambda(z) z p^{\prime}(z),
$$

where the function $\lambda$ is given by (29).

The assumption $G=\mathscr{L} g \in \mathscr{N} \mathscr{K}(\beta)$ is equivalent to

$$
\left(1+\frac{z G^{\prime \prime}(z)}{G^{\prime}(z)}\right)^{-1} \prec \frac{1+(1-2 \beta) z}{1-z} ;
$$

hence there exists a Schwarz function $w$ such that

$$
\left(1+\frac{z G^{\prime \prime}(z)}{G^{\prime}(z)}\right)^{-1}=\frac{1+(1-2 \beta) w(z)}{1-w(z)}, \quad z \in \mathcal{U} .
$$

By simple calculations, we get

$$
\left(2+\frac{z G^{\prime \prime}(z)}{G^{\prime}(z)}\right)^{-1}=\frac{1+(1-2 \beta) w(z)}{2(1-\beta w(z))}, \quad z \in \mathscr{U} ;
$$

that is,

$$
\lambda(z)=\left(2+\frac{z G^{\prime \prime}(z)}{G^{\prime}(z)}\right)^{-1} \prec \frac{1+(1-2 \beta) z}{2(1-\beta z)}=: \phi(z) .
$$


Since the circular transform $\phi$ maps the unit disk $\mathscr{U}$ onto the disk

$$
\begin{aligned}
& \Omega=\{w \in \mathbb{C}:|w-\omega|<r\}, \\
& \quad \text { with } \omega=\frac{1+2 \beta}{2(1+\beta)}, \quad r=\frac{1}{2(1+\beta)},
\end{aligned}
$$

from the subordination property (6) we deduce that the subordination relation (53) is equivalent to

$$
\lambda(z)=u(z)+i v(z) \in \Omega, \quad z \in \mathcal{U} .
$$

With the above notation, the condition (21) becomes

$$
\alpha u(z)-\alpha \gamma|v(z)|-\gamma \geq 0, \quad z \in \mathscr{U} .
$$

We will prove that if $\gamma$ satisfies the assumption (42), that is, $0<\gamma \leq \tilde{\gamma}$, then the subordination (53) implies that the inequality (56) holds, that is,

$$
\Omega \subset \Delta,
$$

where

$$
\Delta=\{w=u+i v \in \mathbb{C}: \alpha u-\alpha \gamma|v|-\gamma \geq 0\} .
$$

Since the sets $\Omega$ and $\Delta$ are symmetric with respect to the real axis, the above inclusion is equivalent to

$$
\widetilde{\Omega} \subset \widetilde{\Delta},
$$

where

$$
\begin{aligned}
& \widetilde{\Omega}=\{w=u+i v \in \Omega: v \geq 0\}, \\
& \widetilde{\Delta}=\{w=u+i v \in \Delta: v \geq 0\} .
\end{aligned}
$$

The line $(d)$ containing the half-line

$$
\alpha u-\alpha \gamma v-\gamma=0, \quad v \geq 0,
$$

which is a part of the boundary the set $\widetilde{\Delta}$, has the equation

$$
\alpha u-\alpha \gamma v-\gamma=0 .
$$

Thus, the line $(d)$ intersects the real axis in the point $\gamma / \alpha$ and contains the point $-i / \alpha$ (see Figure 1 ). The smallest set $\widetilde{\Delta}$ that includes the domain $\widetilde{\Omega}$ is obtained for the case when the line $(d)$, containing the point $-i / \alpha$, is tangent to the upper boundary of $\widetilde{\Omega}$, which is the half-circle

$$
(\mathscr{C})=\left\{w=u+i v \in \mathbb{C}:(u-\omega)^{2}+v^{2}=r^{2}, v \geq 0\right\} .
$$

In this case, as it is shown in the figure, the line $(d)$ becomes the tangent line $\left(d_{\tau}\right)$ to $(\mathscr{C})$.

We will determine now the equation of the tangent line $\left(d_{\tau}\right)$. If we consider the family $\left(d_{m}\right)$ of all the lines containing the point $-i / \alpha$, with nonnegative angular coefficient, that is,

$$
\left(d_{m}\right): v=m u-\frac{1}{\alpha}, \quad m \in(0,+\infty),
$$

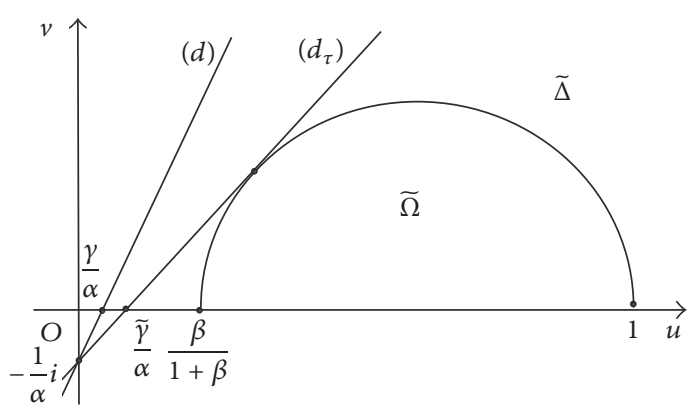

Figure 1: The inclusion $\widetilde{\Omega} \subset \widetilde{\Delta}$.

we will solve the system

$$
\begin{aligned}
(u-\omega)^{2}+v^{2} & =r^{2}, \quad v \geq 0 \\
v & =m u-\frac{1}{\alpha} .
\end{aligned}
$$

It follows that

$$
\begin{aligned}
& \alpha^{2}\left(1+m^{2}\right) u^{2}-2 \alpha(\alpha \omega+m) u+\alpha^{2}\left(\omega^{2}-r^{2}\right)+1 \\
& \quad=0,
\end{aligned}
$$

and the line is tangent to the half-circle if and only if the discriminant of the above quadratic form is zero, that is,

$$
\alpha^{2}\left(\omega^{2}-r^{2}\right) m^{2}-2 \alpha \omega m-\alpha^{2} r^{2}+1=0 .
$$

This last equation has the biggest positive root

$$
\widetilde{m}=\frac{\omega+r \sqrt{\left(\omega^{2}-r^{2}\right) \alpha^{2}+1}}{\alpha\left(\omega^{2}-r^{2}\right)} ;
$$

hence the tangent line to the half-circle $(\mathscr{C})$, which contains the point $-i / \alpha$, has the equation

$$
\left(d_{\tau}\right): v=\widetilde{m} u-\frac{1}{\alpha} .
$$

The tangent $\left(d_{\tau}\right)$ intersects the real axis in the point $1 /(\alpha \widetilde{m})$; hence we deduce that the inclusion (59) holds if and only if

$$
0<\frac{\gamma}{\alpha} \leq \frac{1}{\alpha \widetilde{m}},
$$

that is

$$
\begin{aligned}
0 & <\gamma \leq \frac{1}{\widetilde{m}}=\frac{\alpha\left(\omega^{2}-r^{2}\right)}{\omega+r \sqrt{\left(\omega^{2}-r^{2}\right) \alpha^{2}+1}} \\
& =\frac{2 \alpha \beta}{1+2 \beta+\sqrt{(\beta /(1+\beta)) \alpha^{2}+1}}=\tilde{\gamma}(\alpha, \beta) .
\end{aligned}
$$

Thus, if the parameter $\gamma$ satisfies the assumption (42), then the condition (21) of Lemma 5 is fulfilled, and our result follows from Theorem 6 . 
Putting $g(z)=z$ in Theorem 9, for $\beta \rightarrow 1^{-}$, we get the next result.

Corollary 10. Suppose that $\alpha_{0}$ is defined by (20), and for $0<$ $\alpha \leq \alpha_{0}$ let $\gamma$ satisfy the condition

$$
0<\gamma \leq \frac{2 \alpha}{3+\sqrt{\alpha^{2} / 2+1}} .
$$

If $f \in \mathscr{A}$ and $F=\mathscr{L} f$, then

$$
\left|f^{\prime}(z)\right|<\frac{\pi}{2} \eta(\alpha, \gamma)=\frac{\pi}{2}\left(\alpha+\frac{2}{\pi} \tan ^{-1} \gamma\right), \quad z \in \mathscr{U}
$$

implies

$$
\left|\arg F^{\prime}(z)\right|<\frac{\pi}{2} \alpha, \quad z \in \mathscr{U} .
$$

Example 11. For $f \in \mathscr{A}$ and $F=\mathscr{L} f$, considering the special case $\gamma=2-\sqrt{3}$, from Corollary 10 we deduce the next implication.

$$
\begin{aligned}
& \text { If } \\
& \begin{aligned}
0.54552 \ldots & \simeq \frac{-168+108 \sqrt{3}+164 \sqrt{2}-92 \sqrt{6}}{47} \leq \alpha \\
& \leq \frac{17}{12},
\end{aligned}
\end{aligned}
$$

then

$$
\begin{aligned}
\left|f^{\prime}(z)\right| & <\frac{\pi}{2} \eta(\alpha, 2-\sqrt{3})=\frac{\pi}{2}\left(\alpha+\frac{1}{6}\right), \quad z \in \mathcal{U} \\
& \Downarrow \\
\left|\arg F^{\prime}(z)\right| & <\frac{\pi}{2} \alpha, \quad z \in \mathcal{U} .
\end{aligned}
$$

\section{Competing Interests}

The authors declare that there is no conflict of interests regarding the publication of this paper.

\section{Acknowledgments}

Dr. Badr S. Alkahtani and Dr. Rubayyi Alqahtani extend their sincere appropriations to the Deanship of Scientific Research at King Saud University for funding this Profile Research Group (PRG-1437-35).

\section{References}

[1] M. Nunokawa, S. Owa, J. Nishiwaki, K. Kuroki, and T. Hayami, "Differential subordination and argumental property," Computers \& Mathematics with Applications, vol. 56, no. 10, pp. 27332736, 2008.

[2] V. Ravichandran and S. Sivaprasad Kumar, "Argument estimate for starlike functions of reciprocal order," Southeast Asian Bulletin of Mathematics, vol. 35, no. 5, pp. 837-843, 2011.

[3] C. Pommerenke, Univalent Functions, Vanderhoeck and Ruprecht, Göttingen, Germany, 1975.
[4] S. S. Miller and P. T. Mocanu, Differential Subordinations. Theory and Applications, vol. 225 of Monographs and Textbooks in Pure and Applied Mathematics, Marcel Dekker, New York, NY, USA, 2000.

[5] M. K. Aouf, J. Dziok, and J. Sokól, "On a subclass of strongly starlike functions," Applied Mathematics Letters, vol. 24, no. 1, pp. 27-32, 2011.

[6] N. E. Cho, "Argument estimates of certain meromorphic functions," Communications Korean Mathematical Society, vol. 15, no. 2, pp. 263-274, 2000.

[7] N. E. Cho and J. A. Kim, "Angular estimations of certain analytic functions," Journal of the Korean Mathematical Society, vol. 34, no. 2, pp. 427-436, 1997.

[8] N. E. Cho, Y. C. Kim, and H. M. Srivastava, "Argument estimates for a certain class of analytic functions," Complex Variables. Theory and Application, vol. 38, no. 3, pp. 277-287, 1999.

[9] N. E. Cho, J. Patel, and G. P. Mohapatra, "Argument estimates of certain multivalent functions involving a linear operator," International Journal of Mathematics and Mathematical Sciences, vol. 31, no. 11, pp. 659-673, 2002.

[10] N. E. Cho and H. M. Srivastava, "Argument estimates of certain analytic functions defined by a class of multiplier transformations," Mathematical and Computer Modelling, vol. 37, no. 1-2, pp. 39-49, 2003.

[11] N. E. Cho, S. Y. Woo, and S. Owa, "Argument estimates of certain meromorphic functions," Mathematica Balkanica. New Series, vol. 15, no. 3-4, pp. 285-295, 2001.

[12] S. Kanas and T. Sugawa, "Strong starlikeness for a class of convex functions," Journal of Mathematical Analysis and Applications, vol. 336, no. 2, pp. 1005-1017, 2007.

[13] M. Nunokawa, "On the order of strongly starlikeness of strongly convex functions," Japan Academy. Proceedings. Series A. Mathematical Sciences, vol. 69, no. 7, pp. 234-237, 1993.

[14] M. Nunokawa, S. Owa, H. Saitoh, and N. N. Pascu, "Argument estimates for certain analytic functions," Proceedings of the Japan Academy, Series A, Mathematical Sciences, vol. 79, no. 10, pp. 163-166, 2003.

[15] C. Pommerenke, "On close-to-convex analytic functions," Transactions of the American Mathematical Society, vol. 114, pp. 176-186, 1965.

[16] S. Ponnusamy, "Differential subordinations concerning starlike functions," Proceedings of the Indian Academy of SciencesMathematical Sciences, vol. 104, no. 2, pp. 397-411, 1994.

[17] J. K. Prajapat and S. P. Goyal, "Applications of Srivastava-Attiya operator to the classes of strongly starlike and strongly convex functions," Journal of Mathematical Inequalities, vol. 3, no. 1, pp. 129-137, 2009.

[18] V. Ravichandran, M. Darus, and N. Seenivasagan, "On a criteria for strong starlikeness," Australian Journal of Mathematical Analysis and Applications, vol. 2, no. 1, article 6, 12 pages, 2005.

[19] S. Ponnusamy, "Differential subordination and Bazilević functions," Proceedings of the Indian Academy of Sciences: Mathematical Sciences, vol. 105, no. 2, pp. 169-186, 1995.

[20] R. J. Libera, "Some classes of regular univalent functions," Proceedings of the American Mathematical Society, vol. 16, pp. 755$758,1965$. 


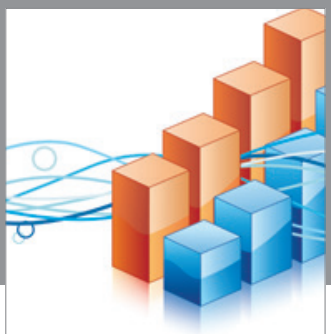

Advances in

Operations Research

vatem alat4

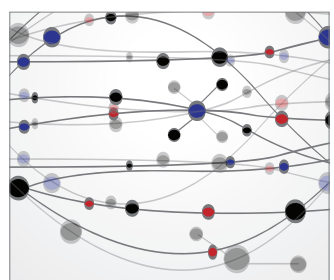

\section{The Scientific} World Journal
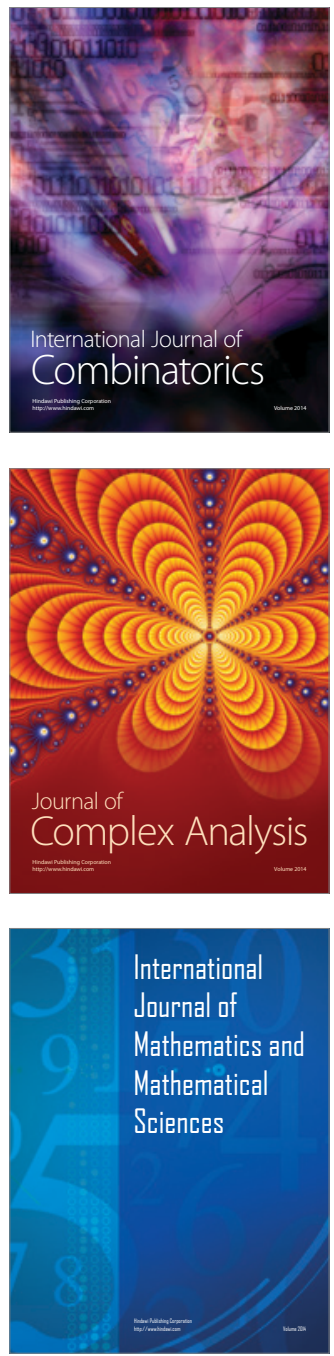
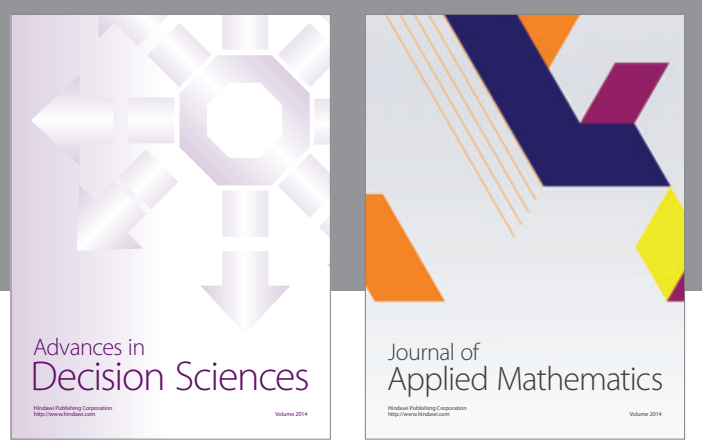

Algebra

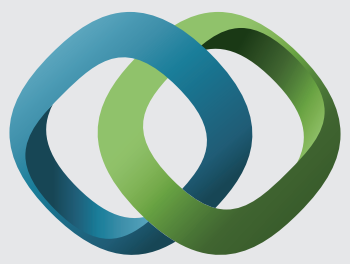

\section{Hindawi}

Submit your manuscripts at

http://www.hindawi.com
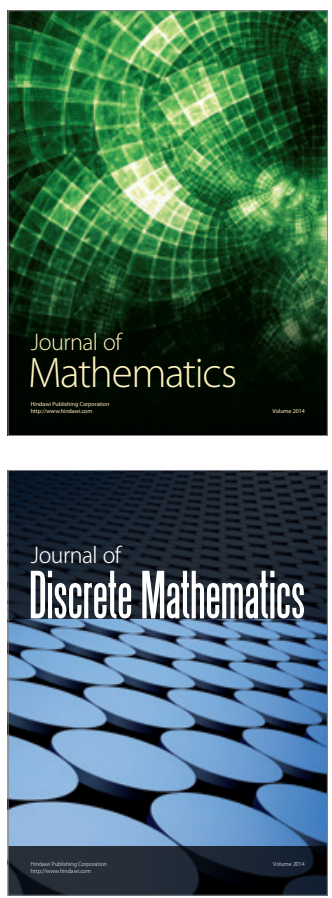

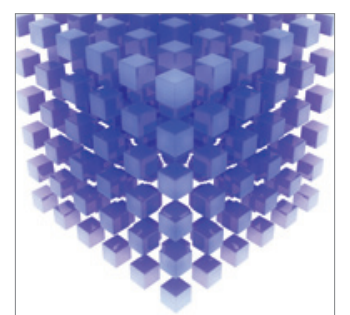

Mathematical Problems in Engineering
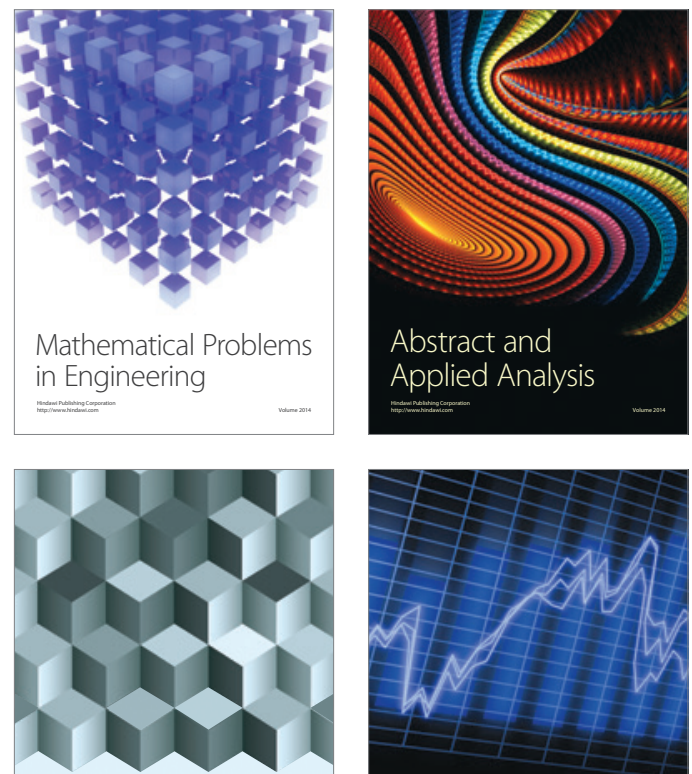

Journal of

Function Spaces

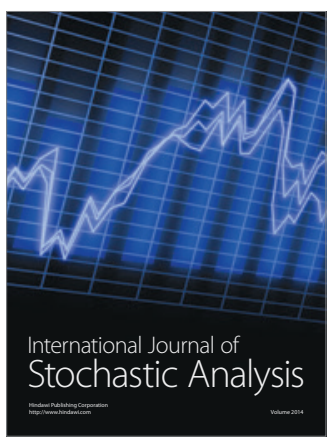

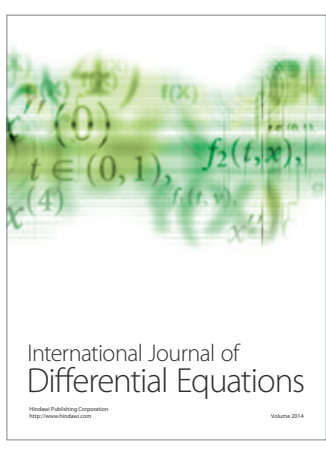
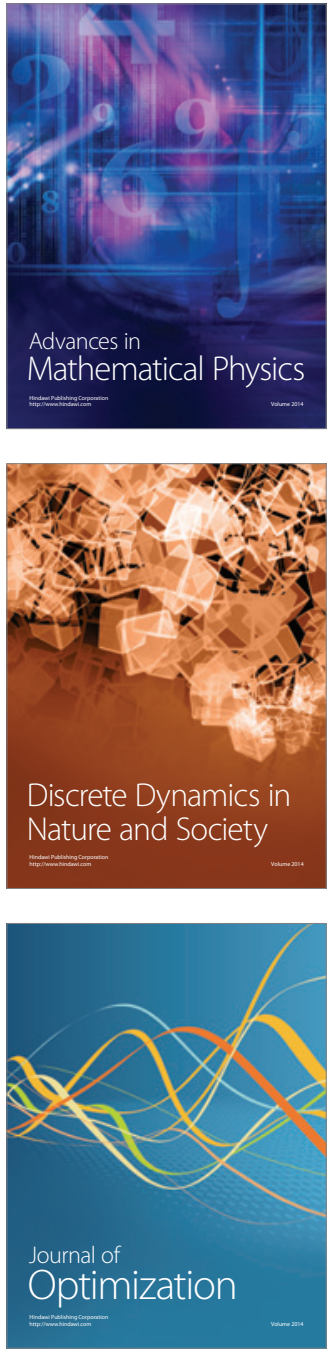